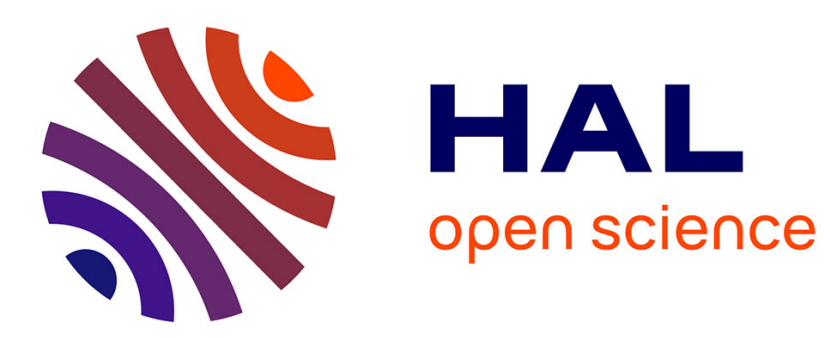

\title{
Les matrices végétales: leurs effets sur la biodisponibilité des caroténoïdes
}

Patrick Borel

\section{To cite this version:}

Patrick Borel. Les matrices végétales: leurs effets sur la biodisponibilité des caroténoïdes. Cahiers de Nutrition et de Diététique, 2018, 53 (2), pp.114-122. 10.1016/j.cnd.2018.02.002 hal-01992015

\section{HAL Id: hal-01992015 https://hal-amu.archives-ouvertes.fr/hal-01992015}

Submitted on 24 Jan 2019

HAL is a multi-disciplinary open access archive for the deposit and dissemination of scientific research documents, whether they are published or not. The documents may come from teaching and research institutions in France or abroad, or from public or private research centers.
L'archive ouverte pluridisciplinaire HAL, est destinée au dépôt et à la diffusion de documents scientifiques de niveau recherche, publiés ou non, émanant des établissements d'enseignement et de recherche français ou étrangers, des laboratoires publics ou privés. 


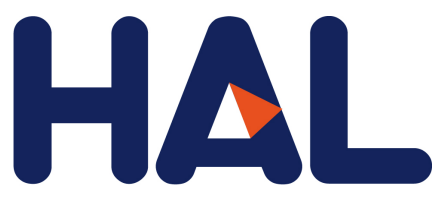

archives-ouvertes

\title{
Les matrices végétales: leurs effets sur la biodisponibilité des caroténoïdes. Fruit and vegetable matrices: their effects on carotenoid bioavailability
}

\author{
Patrick Borel
}

\section{- To cite this version:}

Patrick Borel. Les matrices végétales: leurs effets sur la biodisponibilité des caroténoïdes. Fruit and vegetable matrices: their effects on carotenoid bioavailability. Cahiers de Nutrition et de Diététique, Elsevier Masson, 2018, 53 (2), pp.114-122. <hal-01992015>

\section{HAL Id: hal-01992015 https://hal-amu.archives-ouvertes.fr/hal-01992015}

Submitted on 24 Jan 2019

HAL is a multi-disciplinary open access archive for the deposit and dissemination of scientific research documents, whether they are published or not. The documents may come from teaching and research institutions in France or abroad, or from public or private research centers.
L'archive ouverte pluridisciplinaire HAL, est destinée au dépôt et à la diffusion de documents scientifiques de niveau recherche, publiés ou non, émanant des établissements d'enseignement et de recherche français ou étrangers, des laboratoires publics ou privés. 
Les matrices végétales : leurs effets sur la biodisponibilité des caroténoïdes.

Fruit and vegetable matrices: their effects on carotenoid bioavailability.

Patrick Borel

INRA, INSERM, Aix Marseille Univ, C2VN, Marseille, France

adresse:

$\mathrm{C} 2 \mathrm{VN}$ «Centre de recherches Cardio-Vasculaires et Nutrition de marseille»

UMR 1260 INRA/ 1263 INSERM / Université d'Aix-Marseille

Faculté de Médecine

27, Boulevard Jean-Moulin

13385 Marseille Cedex 5

Tél: (+33) 491324277

E-mail: Patrick.Borel@univ-amu.fr 
Il peut sembler étrange de s'intéresser à l'effet des matrices végétales sur la biodisponibilité des caroténoïdes (CAR) puisque les aliments d'origine végétales sont, de loin, le principal pourvoyeur de ces phytomicronutriments [1] chez l'homme, et de ce fait la biodisponibilité de ces métabolites secondaires des végétaux est obligatoirement affectée par les matrices végétales dans lesquelles ils sont ingérés. Néanmoins, les données disponibles montrent que l'effet de la matrice végétale est très variable suivant les végétaux et suivant l'espèce moléculaire de CAR, e.g. $\beta$-carotène, lutéine ou lycopène. Elles montrent aussi que certains procédés technologiques et culinaires modulent significativement les effets des matrices végétales sur la biodisponibilité des CAR. Comprendre les facteurs qui expliquent les effets des matrices végétales sur la biodisponibilité des CAR est donc un enjeu particulièrement intéressant pour améliorer la biodisponibilité de certains de ces phytomicronutriments qui peuvent être conditionnellement très importants pour la santé humaine. Ainsi, les CAR provitaminiques A, e.g. $\beta$-carotène, $\alpha$-carotène et $\beta$-cryptoxanthine, sont les seuls pourvoyeurs de vitamine A chez les végétaliens, et les principaux pourvoyeurs de cette vitamine chez les végétariens et dans les populations qui sont sujettes à la carence en vitamine A. Par ailleurs, les données s'accumulent pour montrer que certains CAR contribuent à diminuer l'incidence de certaines pathologies, e.g. lycopène et maladies cardiovasculaires [2-5], lutéine et dégénérescence maculaire liée à l'âge [6]. Enfin, il est maintenant bien établi que certains CAR ont des effets biologiques bénéfiques pour la santé, les plus démontrés étant leurs effets antioxydants [2,7] et anti-inflammatoires [8].

\section{Les CAR, des métabolites secondaires des végétaux et des phytomicronutriments}

Les CAR sont qualifiés par les chercheurs en biologie végétale de «métabolites secondaires » des végétaux et par les nutritionnistes de «phytomicronutriments ». Cela vient 
du fait que les premiers s'intéressent à leurs fonctions dans les plantes alors que les seconds s'intéressent à leurs effets sur notre métabolisme et sur notre santé. Le rôle des caroténoïdes dans les plantes sont nombreux et ne sont probablement pas tous élucidés. Ce n'est pas l'objet de cette revue de les décrire en détail mais les principaux sont : un rôle dans la photosynthèse (ils étendent le spectre de lumière qui peut être utilisé par les systèmes photosynthétiques et protègent les chlorophylles en convertissant une partie de l'excès d'énergie en chaleur), un rôle dans la coloration des végétaux, un rôle de précurseur d'hormones comme les strigolactones et l'acide abscissique. La diversité de ces rôles explique la diversité de localisation des CAR dans les cellules végétales (voir chapitre 3), ce qui contribue à expliquer les différences importantes de biodisponibilité des CAR entre les végétaux. Plus de sept cents CAR ont été identifiés chez les êtres vivants, principalement dans le règne végétal, et une cinquantaine d'entre eux et de leurs métabolites a été identifiée dans le sang et dans certains tissus humains. Néanmoins, six CAR ont plus particulièrement été étudiés par les nutritionnistes du fait de leur abondance dans notre alimentation et dans notre organisme. Il s'agit du $\beta$-carotène, du lycopène, de la lutéine, de l' $\alpha$-carotène, de la $\beta$-cryptoxanthine et de la zéaxanthine.

Pour les nutritionnistes les phytomicronutriments (phytochemicals pour les anglophones) sont, comme les racines du nom l'indiquent, des molécules essentiellement ${ }^{1}$ apportées par les aliments d'origine végétale et présentes en faible quantité dans notre alimentation (relativement aux macronutriments). Les phytomicronutriments ne sont pas synthétisés par l'homme et leur présence dans notre organisme provient essentiellement de notre alimentation. Ils sont subdivisés en trois grandes classes : les terpénoïdes (phytostérols,

\footnotetext{
${ }^{1}$ À noter que l'on peut aussi trouver des phytomicronutriments, ou certains de leurs métabolites, dans certains aliments d'origine animale, mais leur présence dans ces aliments provient essentiellement de la consommation par les animaux de végétaux apportant ces composés.
} 
CAR...), les composés phénoliques (flavonoïdes et non-flavonoïdes...) et les composés soufrés (glucosinolates, sulfures d'allyle...). Un ouvrage récent fournira au lecteur intéressé beaucoup plus d'informations concernant ces composés [1]. L'intérêt des nutritionnistes pour les phytomicronutriments vient du fait qu'ils ont des effets biologiques chez l'homme qui, bien que généralement de faible amplitude, sont la plupart du temps bénéfiques pour notre santé à long terme. Ainsi la consommation de CAR est inversement associée à l'incidence de certaines pathologies liées à l'âge [4-6, 9]. De plus certains CAR peuvent être, ou sont à la limite d'être, considérés comme des vitamines. C'est le cas des CAR provitaminiques A, e.g. $\beta$-carotène, $\alpha$-carotène et $\beta$-cryptoxanthine, qui sont essentiels dans certaines populations ${ }^{2}$. C'est presque le cas de la lutéine qui semble avoir un rôle physiologique dans la fonction visuelle. Il a en effet été montré que cette xanthophylle (CAR possédant des groupements oxygénés) améliore les performances visuelles et la sensibilité au contraste [10-16]. Ce n'est pas l'objet de cette revue de faire un état de l'art de tous les effets biologiques que les CAR semblent exercer chez l'homme. Ceux-ci sont en effet nombreux et certains ne sont pas suffisamment établis. Ces effets justifient néanmoins d'étudier leur biodisponibilité chez l'homme, d'autant plus que celle-ci s'avère très faible, très variable, et est affectée par de nombreux facteurs $[17,18]$.

\section{Sources alimentaires de CAR}

Dans la mesure où les CAR ne sont pas synthétisés par les animaux et que seule une partie des CAR ingérés par les animaux de rente est stockée dans leurs tissus, les principales sources de CAR dans notre alimentation sont les aliments d'origine végétale, et plus particulièrement les fruits et légumes. On devrait plutôt dire les légumes et les fruits car,

\footnotetext{
${ }^{2}$ Chez les végétaliens pour lesquels c'est la seule source de vitamine A ou dans des populations qui consomment très peu d'aliments d'origine animale pour des raisons socio-économiques.
} 
d'après des données Espagnoles $[19,20], 84 \%$ du $\beta$-carotène est apporté par les légumes et $5 \%$ par les fruits, $86 \%$ du lycopène est apporté par les légumes et $11 \%$ par les fruits, et $84 \%$ de la lutéine est apportée par les légumes et 5\% par les fruits. A noter toutefois que cette répartition dépend de ce que l'on classe comme fruit ou légume. Le lycopène est en effet essentiellement apporté par la tomate, et ses produits dérivés, qui est considérée comme un légume dans les enquêtes alimentaires alors qu'il s'agit bien du fruit de la plante. Quoi qu'il en soit, les aliments d'origine animale n'apportent que $14 \%$ de la lutéine, $2 \%$ du $\beta$-carotène et moins de $1 \%$ du lycopène. Les interactions des CAR avec la matrice alimentaire se font donc dans un premier temps essentiellement avec les matrices végétales. Il ne faut néanmoins pas oublier que des aliments d'origine végétale et animale sont la plupart du temps consommés dans les mêmes repas et qu'il peut y avoir des interactions entre des CAR provenant des matrices végétales et des composants des matrices animales dans le tube digestif. La plus connue de ces interactions étant celle des CAR avec les lipides des aliments d'origine animale (cf. chapitre 4.2). Une étude clinique a d'ailleurs suggéré que des lipides d'origine animale permettaient une meilleure biodisponibilité du $\beta$-carotène que des lipides d'origine végétale $[21]$.

\section{Localisation et état physique des CAR dans les cellules végétales}

La localisation intracellulaire et l'état physique des CAR dans les aliments d'origine animale et végétale sont très différents [22]. Il est maintenant bien démontré que ces paramètres sont des facteurs clefs qui affectent la biodisponibilité des CAR et il est supposé qu'une meilleure connaissance de ces paramètres pourrait permettre d'identifier des sources végétales comportant des CAR très biodisponibles [22]. Les CAR sont des composés très hydrophobes. Par conséquent, quand ils sont à forte concentration dans un milieu aqueux, ils ont tendance à s'agréger jusqu'à former des cristaux. Par contre, quand ils sont à faible 
concentration ils peuvent s'incorporer dans des structures lipidiques (triglycérides ou films de phospholipides) ou s'associer à certaines protéines de manière plus ou moins spécifique. Ils peuvent aussi interagir avec les polysaccharides qui constituent les parois des cellules végétales. Comme toutes les cellules végétales n'ont pas la même organisation ni la même composition, et comme les CAR peuvent avoir des fonctions biologiques très différentes, on comprend que la localisation intracellulaire des CAR est très variable entre les cellules de différentes plantes, et même entre les cellules de différents tissus d'une même plante (Figure 1). Par ailleurs, l'interaction des CAR avec les différentes structures moléculaires intracellulaires dépend des propriétés physico-chimiques des CAR. Ainsi l'insertion des caroténoïdes dans les membranes cellulaires dépend notamment de leur interaction avec les phospholipides, de leur conformation tridimensionnelle et de la présence de groupements polaires (e.g. fonctions hydroxyles de la lutéine) [23]. A ce jour, six grands types de structures intracellulaires dans lesquelles se retrouvent les CAR ont été décrites [24]. Par ailleurs, les CAR se retrouvent généralement dans plusieurs structures différentes dans la même cellule. Ainsi, dans les légumes verts, e.g. les épinards et les brocolis, les CAR sont préférentiellement localisés dans les complexes macromoléculaires pigments-protéines des photosystèmes (Figure 1A). Ces derniers étant eux-mêmes insérés dans les membranes des thylakoïdes des chloroplastes. Les chloroplastes sont par ailleurs parfois regroupés et entourés d'une membrane rigide et stable. Cet emboitement en «poupées russes» explique que la biodisponibilité des CAR des légumes verts soit plus faible que celle des autres légumes. Les légumes verts, qui peuvent contenir des quantités relativement importantes de $\beta$-carotène, sont ainsi une très mauvaise source de vitamine A [25-27]. Dans les fruits et légumes de couleur rouge, orange ou jaune, les CAR sont préférentiellement localisés dans des chromoplastes. Mais, alors que la morphologie des chloroplastes est relativement bien conservée, celle des chromoplastes est très variable. Il existe ainsi des chromoplastes globulaires, tubulaires, 
membraneux et de type cristallin, et l'état physique des CAR dans ces chromoplastes est lui aussi très variable. On trouve ainsi des CAR sous forme de cristaux solides dans les chromoplastes des tomates et des carottes (Figure 1B), et des CAR sous forme de cristaux liquides dans les chromoplastes globulo-tubulaires de certains fruits oranges/jaunes comme les mangues et les papayes (Figure 1C). Une étude récente a montré que le tout-trans lycopène solubilisé dans une phase lipidique et sous forme de cristaux liquides dans les chromoplastes globulaires des tomates tangerines (Figure 1D) est deux fois plus biodisponible que le lycopène sous forme de cristaux solides dans les chromoplastes cristalloïdes de la tomate commune [28].

\section{Facteurs affectant la biodisponibilité des CAR}

Du fait que la biodisponibilité des CAR peut être très faible, e.g. quelques pourcents du lycopène des tomates crues sont biodisponibles, et est très variable $[17,18]$, et du fait des preuves qui s'accumulent sur l'intérêt santé de certains CAR, de nombreuses recherches ont été, et sont encore, effectuées pour identifier les facteurs qui modulent la biodisponibilité de ces composés [17]. Il ressort de ces recherches que ces facteurs sont très variés. Cela va en effet de l'effet de l'espèce moléculaire du CAR à l'effet des variations génétiques qui modulent l'activité/l'expression des protéines impliquées dans leur biodisponibilité. Ce n'est pas l'objet de cette revue de décrire en détail les effets de ces différents facteurs et le lecteur intéressé pourra trouver les réponses à ses questions dans deux revues récentes sur le sujet $[17,18]$. Nous nous focaliserons sur deux facteurs qui sont liés, directement ou indirectement, à l'effet des matrices végétales sur la biodisponibilité des CAR [17, 29, 30]. Le premier de ces facteurs est l'effet de la localisation intracellulaire et de l'état physique des CAR qui peuvent être très différents dans différentes matrices végétales. Le second facteur est du à l'effet des 
composés constitutifs de la matrice qui activent, ou au contraire inhibent, l'absorption des

\section{CAR (Figure 2).}

\subsection{La matrice alimentaire}

Quand on parle des effets de la matrice alimentaire, on s'intéresse aux effets de facteurs très variés. Le premier facteur est lié à la localisation intracellulaire des CAR. Le second, qui est fortement dépendant du premier, est lié à l'état physique des CAR dans les structures intracellulaires, par exemple sous forme de cristaux solides ou associés à des protéines. Par ailleurs, comme les traitements technologiques et culinaires des aliments peuvent altérer profondément les cellules végétales, et donc la localisation intracellulaire et l'état physique des CAR, on inclut aussi dans les effets de la matrice les effets des traitements de la matrice sur la biodisponibilité des CAR. Il faut enfin noter qu'il y a aussi souvent un chevauchement entre l'effet de la matrice alimentaire et l'effet des effecteurs d'absorption. Par exemple des interactions des CAR avec les polysaccharides des parois végétales, i.e. les fibres alimentaires, peuvent apparaitre lors de la destruction des cellules végétales par les traitements technologiques ou culinaires des aliments. Quoi qu'il en soit, de nombreuses études ont montré que la matrice alimentaire a un effet très significatif sur la bioaccessibilité ${ }^{3}$ et la biodisponibilité de certains CAR [27, 31-36].

Comme décrit dans le chapitre 3, l'état physique et la localisation intracellulaire des CAR dans les végétaux sont très variables (Figure 1). Il a été proposé que cette variabilité est à l'origine des différences très importantes de biodisponibilité des CAR dans divers fruits et légumes [22]. C'est la théorie dite des barrières structurales. Selon cette théorie la biodisponibilité d'un CAR serait inversement liée aux nombres de «barrières »

\footnotetext{
${ }^{3}$ Fraction de CAR qui est extraite de sa matrice alimentaire pour être solubilisée dans les micelles au cours de la digestion. Il s'agit d'une étape clef de la biodisponibilité car on admet que seule la fraction bioaccessible est absorbée.
} 
physiologiques qu'il a à franchir pour atteindre le «véhicule » physiologique qui permettra son absorption, i.e. les micelles mixtes. Ainsi, le lycopène sous forme de cristaux solides dans la cellule de tomates (Figure 1A) devra être libéré de cette cellule, lors de sa digestion et éventuellement aussi de son traitement technologique ou culinaire, puis être mis en contact avec des molécules permettant sa solubilisation dans les micelles, à priori les lipides et les sels biliaires des micelles, pour être efficacement absorbé. On explique ainsi la très mauvaise biodisponibilité du lycopène des tomates quand elles sont consommées crues. Ceci explique aussi que le lycopène des tomates transformées, e.g. sous forme de purée ou de ketchup, est nettement plus bioaccessible [32] et biodisponible [37-39] que le lycopène des tomates crues. D'autres études ont confirmé les différences attendues de bioaccessibilité [32] et de biodisponibilité des CAR quand ils sont situés dans des matrices végétales différentes [27, 28, 40]. Une synthèse des données disponibles suggère que la biodisponibilité des CAR se classe de la façon suivante : fruits avec des cristaux liquides de CAR (exemples : mangues et papayes) > fruits et légumes avec des cristaux solides de CAR (exemples : tomates et carottes) > légumes verts avec des CAR dans les chloroplastes (exemples : épinards et salades). Cette classification est toutefois très grossière et est affectée par de nombreux facteurs, dont le traitement technologique et culinaire des aliments et l'espèce moléculaire de CAR. On a ainsi observé que la bioaccessibilité de la lutéine, pour des raisons encore mal expliquées, est très peu affectée par la nature de la matrice végétale et par son traitement technologique [32]. En cohérence avec la théorie des barrières structurales, il a aussi été montré que la maturation des fruits améliore la biodisponibilité des CAR [41]. On suppose que le ramollissement des fruits facilite la destruction mécanique et enzymatique des barrières structurales lors de la digestion. Bien que la théorie des barrières structurales soit séduisante pour expliquer les différences de biodisponibilité entre différentes matrices végétales, d'autres facteurs sont sans doute impliqués. Il est ainsi probable que l'isomérisation cis de certains 
CAR, qui peut être naturelle dans certaines matrices végétales [28], ou qui peut être induite par les traitements technologiques ou culinaires des aliments [42, 43], soit parfois impliquée dans les différences de biodisponibilité observées entre certaines matrices végétales. Il a en effet été montré que les isomères cis du lycopène sont plus biodisponibles que l'isomère tout trans $[28,44,45]$. C'est ce qui expliquerait la très forte biodisponibilité du lycopène des tomates tangerines par rapport à celle des tomates communes [28]. Un autre facteur confondant à celui des barrières structurales est la présence, dans les matrices végétales, de divers effecteurs d'absorption (cf. chapitre 4.2). L'un de ces effecteurs est toujours présent dans les matrices végétales puisqu'il s'agit des polysaccharides qui constituent la paroi des cellules végétales. Or, il est supposé que ces polysaccharides, appelés fibres alimentaires par les nutritionnistes, complexent une partie des sels biliaires et/ou des CAR et empêchent ainsi leur absorption. Par ailleurs la nature de ces polysaccharides et leur proportion relative varient d'une espèce végétale à l'autre, et on sait très peu de choses sur l'effet relatif de chacun de ces polysaccharides sur la biodisponibilité des CAR. Enfin, d'autres effecteurs peuvent être présents dans certaines matrices végétales et affecter positivement ou négativement la biodisponibilité des CAR (voir chapitre suivant).

\subsection{Les effecteurs d'absorption}

On désigne par la des molécules, ou des assemblages de molécules, qui peuvent soit augmenter soit diminuer l'absorption des CAR.

Parmi ces effecteurs seuls les lipides, et plus précisément les triglycérides, sont connus pour augmenter l'absorption des CAR. Ces macronutriments sont présents en faible quantité dans la plupart des fruits et légumes, à l'exception de certains d'entre eux comme les avocats, mais ils peuvent être apportés en quantité significative quand on consomme des légumes avec, par exemple, un assaisonnement riche en huiles végétales. 
L'effet favorable des lipides vis-à-vis de la biodisponibilité des CAR a été observé dans plusieurs études cliniques [46-49] et dans de nombreuses études chez l'animal. De plus il a été montré qu'une quantité relativement faible de lipides (6 g/repas) suffisait à augmenter significativement la biodisponibilité. Néanmoins une dose supérieure (28 g) augmentait encore plus la biodisponibilité [46] et on ne sait finalement toujours pas quel est le compromis optimal entre l'effet bénéfique d'augmenter la quantité de lipides pour améliorer la biodisponibilité des CAR et l'effet négatif d'augmenter cette quantité vis-à-vis des problèmes de santé que cela peut potentiellement entrainer. Plusieurs mécanismes seraient impliqués. Le premier serait de contribuer à solubiliser une partie des cristaux solides de CAR que l'on retrouve dans les tomates et les carottes par exemple [50]. Le second serait dû à la capacité des lipides à présenter aux CAR une phase hydrophobe dans laquelle ils peuvent se solubiliser [51]. Un transfert de CAR a d'ailleurs été observé entre des matrices végétales et des lipides dans l'estomac humain [52]. Le troisième mécanisme par lequel les lipides favoriseraient la biodisponibilité des CAR est leur capacité à faire sécréter la bile. Celle-ci contient en effet des sels biliaires et des phospholipides qui permettent la constitution de micelles dans la lumière intestinale. Or, les micelles, et plus précisément les micelles mixtes qui sont constituées des lipides biliaires, auxquels s'associent les produits de la digestion des triglycérides alimentaires (acides gras et monoglycérides), sont les structures qui solubilisent les CAR dans le tube digestif et qui permettent leur transport jusqu'à l'entérocyte (Figure 2).

Il existe plusieurs Effecteurs qui diminuent 1'absorption des CAR (Figure 2). Le premier, en masse, est les fibres alimentaires. Ces polysaccharides interagissent avec de nombreuses molécules présentes dans la lumière intestinale au cours de la digestion. On a ainsi montré qu'ils peuvent adsorber des sels biliaires et du cholestérol, ce qui diminue leur absorption et ce qui explique en partie leurs effets hypocholestérolémiants. Il est ainsi supposé que ces polymères, ou du moins certains d'entre eux, diminuent la biodisponibilité des CAR 
$[53,54]$, probablement en affectant leur micellisation [55]. Un autre effecteur qui semble diminuer l'absorption des CAR sont les phytostérols. Ces composés, comme leur nom l'indique, sont des stérols présents dans les cellules végétales. De nombreuses études ont permis de démontrer qu'ils diminuent l'absorption du cholestérol, le mécanisme principal serait leur capacité à entrer en compétition avec ce dernier quant à l'incorporation dans les micelles. On suppose que le même mécanisme explique leur capacité à inhiber la biodisponibilité de certains CAR [56-58]. D’autres composés présents dans les matrices végétales pourraient diminuer l'absorption des CAR mais très peu de données sont disponibles. On peut ainsi mentionner l'effet inhibiteur d'un composé phénolique sur l'absorption d'un CAR [59], mais cela reste à confirmer et l'effet potentiel d'autres phytomicronutriments reste à étudier.

\subsection{Effets des traitements technologiques et culinaires des matrices végétales sur la} biodisponibilité des CAR

L'effet des traitements technologiques et culinaires des aliments sur la biodisponibilité des CAR est intrinsèquement lié aux caractéristiques de la matrice alimentaire car ces traitements vont profondément modifier la structure de cette matrice et donc la répartition intracellulaire et l'état physique des CAR. Ils vont aussi créer des interactions entre les CAR et des composants de la matrice végétale qui ne sont pas naturellement en contact quand celleci est intacte. L'effet de ces traitements a été énormément étudié et il n'est pas possible de citer l'ensemble des articles sur ce sujet. Le lecteur intéressé pourra se référer à la sélection suivante [43, 60-64]. Compte tenu du fait que les CAR sont des molécules très labiles, on a tout d'abord supposé que ces traitements ont un effet négatif sur la biodisponibilité des CAR en détruisant une grande partie d'entre eux [65-69]. Néanmoins on s'est aperçu que, bien qu'ils soient très labiles quand ils sont purifiés dans des solvants organiques, les CAR sont 
généralement protégés de la dégradation dans les matrices végétales du fait de leur association avec des lipides ou des protéines, ou quand ils sont sous forme de cristaux solides. On a aussi supposé que ces traitements pussent avoir une effet négatif sur la biodisponibilité des CAR en créant de nouvelles interactions entres les CAR et certains composants des cellules végétales. On a enfin supposé que ces traitements pouvaient au contraire avoir un effet positif sur la biodisponibilité en cassant les cellules végétales, ce qui devrait permettre aux CAR de franchir plus facilement les différentes barrières structurales qui les séparent des micelles mixtes, ou en isomérisant une fraction des CAR en isomères cis [70, 71]. Malheureusement, les nombreuses études sur ce sujet se sont contentées de mesurer l'effet global des traitements culinaires et technologiques sur la biodisponibilité. En résumé, l'effet des traitements mécaniques et enzymatiques est plutôt favorable à la biodisponibilité [22] (Figure 2) ce qui suggère que l'effet bénéfique sur les barrières structurales l'emporte sur les effets négatifs, i.e. les nouvelles interactions créées par ces traitements avec des effecteurs d'absorption négatifs, e.g. les fibres alimentaires. L'effet du traitement thermique est moins évident. On peut supposer que l'amélioration de la biodisponibilité par, par exemple, l'isomérisation cis des CAR sous l'effet de la chaleur est compensée par l'effet négatif engendré par la destruction d'une fraction des CAR.

6. Recommandations visant à améliorer la biodisponibilité des CAR présents dans les matrices végétales

Comme la biodisponibilité des CAR présents dans certaines matrices végétales, e.g. les légumes verts, peut-être très faible, de nombreuses études ont été consacrées à la façon d'améliorer cette biodisponibilité. En résumé trois stratégies peuvent être proposées. La première est de conseiller la consommation de certains fruits qui ont des CAR plus 
biodisponibles que certains légumes. Par exemple, recommander la consommation de fruits riches en $\beta$-carotène plutôt que des légumes à feuilles vertes riches en ce même CAR provitaminique A. La seconde est de recommander de consommer les végétaux contenant des CAR peu biodisponibles, e.g. les légumes à feuilles vertes, au cours des repas. Ces derniers apportent en effet généralement une quantité suffisante de lipides pour favoriser l'extraction et la micellisation des CAR de ces végétaux. Ces stratégies sont particulièrement intéressantes pour favoriser l'assimilation des CAR provitaminiques A dans les pays où sévit la carence en vitamine A [48]. La troisième stratégie est de conseiller de cuire les légumes de façon à diminuer les barrières structurales et à favoriser la libération des CAR de leur matrice végétale [39, 64, 72]. Les effets de différentes méthodes de cuisson des aliments ont été étudiés [61, 73-75], mais aucun consensus ne ressort quant à la méthode optimale de cuisson vis-à-vis de la biodisponibilité des CAR. D’autres stratégies ont enfin été envisagées comme l'ingestion simultanée d'aliments favorisant l'isomérisation cis de certains CAR, ce qui devrait théoriquement faciliter leur absorption [71], néanmoins elles restent anecdotiques.

En conclusion, cette revue de la littérature montre que les matrices végétales sont un des facteurs qui affectent le plus la biodisponibilité des CAR. Les mécanismes impliqués sont très variés, cela va de l'état physique des CAR et de leur localisation dans les cellules végétales à leurs interactions potentielles avec des composants de la matrice végétale. Certains de ces composants sont capables d'améliorer la biodisponibilité des CAR, e.g. les lipides, alors que d'autres ont plutôt un effet inverse, e.g. les fibres alimentaires. Ces connaissances fondamentales permettent de mieux comprendre l'effet des traitements technologiques et culinaires des matrices végétales sur la biodisponibilité des CAR. Elles permettent aussi de proposer des choix alimentaires et/ou des traitements technologiques et/ou 
culinaires des aliments visant à améliorer la biodisponibilité de ces phytomicronutriments qui peuvent être essentiels pour certaines populations.

A retenir : les médecins nutritionnistes, les diététiciens et les professionnels de l'industrie agro-alimentaire connaissent le rôle provitaminique de certains caroténoïdes (CAR) et sont maintenant au fait de l'intérêt de certains autres CAR, e.g. lutéine et lycopène, pour la santé. Ils savent aussi en général que la biodisponibilité de ces phytomicronutriments est relativement faible et peut être améliorée. Ils sont généralement moins informés du fait que la biodisponibilité des CAR présents dans les différents fruits et légumes est très variable. Ils connaissent enfin très peu les mécanismes responsables de cette variabilité, e.g. la variabilité de l'état physique et de la localisation intracellulaire des CAR dans les différents végétaux. Ils sont donc en attente de recommandations, nutritionnelles ou relatives aux transformations industrielles ou culinaires, permettant d'améliorer la biodisponibilité de ces phytomicronutriments. Les données actuelles permettent de proposer trois stratégies pour contrecarrer l'effet inhibiteur des matrices végétales sur la biodisponibilité des CAR. La première c'est de les consommer de préférence dans des fruits. La seconde c'est de les consommer avec un minimum de lipides ( $>6 \mathrm{~g})$. La troisième c'est de consommer les légumes de préférence cuits. Ces stratégies sont particulièrement intéressantes dans le cas de la lutte contre la carence en vitamine A mais peuvent aussi être intéressantes pour améliorer la biodisponibilité de CAR impliqués dans la prévention de certaines pathologies dégénératives. 


\section{Résumé :}

Alors que les fruits et légumes sont, de loin, notre principale source de caroténoïdes (CAR), la localisation intracellulaire et l'état physique des CAR dans les cellules végétales, ainsi que des interactions avec certains composants de ces aliments, affectent significativement la biodisponibilité des CAR. La bioaccessibilité (i.e. l'incorporation dans les micelles) est en effet affectée par la localisation des CAR dans les différents plastides présents dans les cellules végétales, e.g. chloroplastes, chromoplastes, et par l'état physique des CAR dans ces plastides, e.g. sous forme crystalline ou associés à des protéines. La bioaccessibilité des CAR peut par ailleurs être diminuée par l'interaction des CAR, ou des sels biliaires des micelles, avec certaines molécules présentes dans les matrices végétales, e.g. fibres et phytostérols. Elle peut par contre être augmentée par les triglycérides présents dans certains fruits et légumes, e.g. avocats, ou dans d'autres aliments. La compréhension des mécanismes moléculaires qui expliquent les effets des matrices végétales sur la biodisponibilité des CAR permet de proposer des recommandations nutritionnelles et/ou des traitements technologiques ou culinaires visant à optimiser la biodisponibilité de ces phytomicronutriments à effets santé.

Mots clés : état physique des caroténoïdes, localisation intracellulaire des caroténoïdes, traitements technologiques et culinaires des fruits et légumes, lipides, fibres. 


\begin{abstract}
:
Although fruits and vegetables are, by far, our main source of carotenoids (CAR), the intracellular localization and the physical state of CAR in plant cells, as well as interactions with some components of these foods, significantly impair the bioavailability of CAR. The bioaccessibility, i.e. the incorporation of CAR into micelles, is indeed modulated by CAR localisation in the different plastids, e.g. chloroplasts, chromoplasts, as well as by their physical state in these plastids, e.g. as crystals or associated with proteins. CAR bioaccessibility can also be impaired by interactions of CAR, or of bile salts that constitute micelles, with some compounds present in vegetable matrices, e.g. fibers and phytosterols. Conversely, it can be improved by triglycerides present in some fruit and vegetables, e.g. avocados, or in other foods. Deciphering the molecular mechanisms that explain the effect of fruit and vegetable matrices on CAR bioavailability allow to suggest dietary recommandations or food processing or culinary methods that aim to improve the bioavailability of these phytochemicals with some well-established health effects.
\end{abstract}

Key words: physical state of carotenoids, intracellular localization of carotenoids, food processing and culinary processes, lipids, fibers. 
Remerciements : cette revue a été rédigée suite à une communication orale aux JFN de Nantes (13-15 décembre 2017) et à une invitation par le comité de rédaction des cahiers de nutrition et diététique (Eric Bertin rédacteur en chef et Jean-Michel Chardigny, président du comité de rédaction). Merci à Quentin et à Sonia pour leur relecture critique du manuscrit.

Conflits d'intérêt : l'auteur n'a aucun conflit d'intérêt à déclarer. 


\section{Références :}

[1] Amiot MJ, Coxam V, Strigler F. Les phytomicronutriments. Paris : Tec \& Doc Lavoisier; 2012: p. 1-386.

[2] Müller L, Caris-Veyrat C, Lowe G, Bohm V. Lycopene and Its Antioxidant Role in the Prevention of Cardiovascular Diseases - A Critical Review. Crit Rev Food Sci Nutr 2016;56:1868-79.

[3] Mordente A, Guantario B, Meucci E, Silvestrini A, Lombardi E, Martorana GE, et al. Lycopene and cardiovascular diseases: an update. Curr Med Chem 2011;18:1146-63.

[4] Cheng HM, Koutsidis G, Lodge JK, Ashor A, Siervo M, Lara J. Tomato and lycopene supplementation and cardiovascular risk factors: A systematic review and meta-analysis. Atherosclerosis 2017;257:100-8.

[5] Song B, Liu K, Gao Y, Zhao L, Fang H, Li Y, et al. Lycopene and risk of cardiovascular diseases: A meta-analysis of observational studies. Mol Nutr Food Res 2017;61:doi: 10.1002/mnfr.201601009.

[6] Chew EY, Clemons TE, Sangiovanni JP, Danis RP, Ferris FL, 3rd, Elman MJ, et al. Secondary Analyses of the Effects of Lutein/Zeaxanthin on Age-Related Macular Degeneration Progression: AREDS2 Report No. 3. JAMA Ophthalmol 2014;132:142-9.

[7] Kelkel M, Schumacher M, Dicato M, Diederich M. Antioxidant and anti-proliferative properties of lycopene. Free Radic Res 2011;45:925-40.

[8] Marcotorchino J, Romier B, Gouranton E, Riollet C, Gleize B, Malezet-Desmoulins C, et al. Lycopene attenuates LPS-induced TNF-alpha secretion in macrophages and inflammatory markers in adipocytes exposed to macrophage-conditioned media. Mol Nutr Food Res 2012;56:725-32.

[9] Johnson EJ. A possible role for lutein and zeaxanthin in cognitive function in the elderly. Am J Clin Nutr 2012;96:1161S-5S.

[10] Stringham JM, O'Brien KJ, Stringham NT. Contrast Sensitivity and Lateral Inhibition Are Enhanced With Macular Carotenoid Supplementation. Invest Ophthalmol Vis Sci 2017;58:2291-5.

[11] Stringham JM, Hammond BR. Macular pigment and visual performance under glare conditions. Optom Vis Sci 2008;85:82-8.

[12] Ma L, Yan SF, Huang YM, Lu XR, Qian F, Pang HL, et al. Effect of lutein and zeaxanthin on macular pigment and visual function in patients with early age-related macular degeneration. Ophthalmology 2012;119:2290-7. 
[13] Ma L, Lin XM, Zou ZY, Xu XR, Li Y, Xu R. A 12-week lutein supplementation improves visual function in Chinese people with long-term computer display light exposure. Br J Nutr 2009;102:186-90.

[14] Loughman J, Nolan JM, Howard AN, Connolly E, Meagher K, Beatty S. The impact of macular pigment augmentation on visual performance using different carotenoid formulations. Invest Ophthalmol Vis Sci 2012;53:7871-80.

[15] Yao Y, Qiu QH, Wu XW, Cai ZY, Xu S, Liang XQ. Lutein supplementation improves visual performance in Chinese drivers: 1-year randomized, double-blind, placebocontrolled study. Nutrition 2013;29:958-64.

[16] Hammond BR, Fletcher LM, Roos F, Wittwer J, Schalch W. A double-blind, placebocontrolled study on the effects of lutein and zeaxanthin on photostress recovery, glare disability, and chromatic contrast. Invest Ophthalmol Vis Sci 2014;55:8583-9.

[17] Desmarchelier C, Borel P. Overview of carotenoid bioavailability determinants: from dietary factors to host genetic variations. Trends Food Sci Technol 2017;69:270-80.

[18] Bohn T, Desmarchelier C, Dragsted LO, Nielsen CS, Stahl W, Ruhl R, et al. Host-related factors explaining interindividual variability of carotenoid bioavailability and tissue concentrations in humans. Mol Nutr Food Res 2017;61:doi: 10.1002/mnfr.201600685.

[19] Estevez-Santiago R, Beltran-de-Miguel B, Olmedilla-Alonso B. Assessment of dietary lutein, zeaxanthin and lycopene intakes and sources in the Spanish survey of dietary intake (2009-2010). Int J Food Sci Nutr 2016;67:305-13.

[20] Beltran-de-Miguel B, Estevez-Santiago R, Olmedilla-Alonso B. Assessment of dietary vitamin A intake (retinol, alpha-carotene, beta-carotene, beta-cryptoxanthin) and its sources in the National Survey of Dietary Intake in Spain (2009-2010). Int J Food Sci Nutr 2015;66:706-12.

[21] Hu XX, Jandacek RJ, White WS. Intestinal absorption of beta-carotene ingested with a meal rich in sunflower oil or beef tallow: postprandial appearance in triacylglycerol-rich lipoproteins in women. Am J Clin Nutr 2000;71:1170-80.

[22] Schweiggert RM, Carle R. Carotenoid deposition in plant and animal foods and its impact on bioavailability. Crit Rev Food Sci Nutr 2017;57:1807-30.

[23] Lancrajan I, Diehl HA, Socaciu C, Engelke M, ZornKruppa M. Carotenoid incorporation into natural membranes from artificial carriers: liposomes and beta-cyclodextrins. Chem Phys Lipids 2001;112:1-10. 
[24] Egea I, Barsan C, Bian W, Purgatto E, Latche A, Chervin C, et al. Chromoplast differentiation: current status and perspectives. Plant Cell Physiol 2010;51:1601-11.

[25] de Pee S, West CE, Muhilal, Karyadi D, Hautvast JG. Lack of improvement in vitamin A status with increased consumption of dark-green leafy vegetables. Lancet 1995;346:7581.

[26] Micozzi MS, Brown ED, Edwards BK, Bieri JG, Taylor PR, Khachik F, et al. Plasma carotenoid response to chronic intake of selected foods and beta- carotene supplements in men. Am J Clin Nutr 1992;55:1120-5.

[27] van het Hof KH, Tijburg LBM, Pietrzik K, Weststrate JA. Influence of feeding different vegetables on plasma levels of carotenoids, folate and vitamin C. Effect of disruption of the vegetable matrix. Br J Nutr 1999;82:203-12.

[28] Cooperstone JL, Ralston RA, Riedl KM, Haufe TC, Schweiggert RM, King SA, et al. Enhanced bioavailability of lycopene when consumed as cis-isomers from tangerine compared to red tomato juice, a randomized, cross-over clinical trial. Mol Nutr Food Res 2015;59:658-69.

[29] Borel P. Factors affecting intestinal absorption of highly lipophilic food microconstituents (fat-soluble vitamins, carotenoids and phytosterols). Clin Chem Lab Med 2003;41:979-94.

[30] West CE, Castenmiller JJJM. Quantification of the "SLAMENGHI" factors for carotenoid bioavailability and bioconversion. Internat J Vit Nutr Res 1998;68:371-7.

[31] Castenmiller JJM, West CE, Linssen JPH, van het Hof KH, Voragen AGJ. The food matrix of spinach is a limiting factor in determining the bioavailability of beta-carotene and to a lesser extent of lutein in humans. J Nutr 1999;129:349-55.

[32] Reboul E, Richelle M, Perrot E, Desmoulins-Malezet C, Pirisi V, Borel P. Bioaccessibility of carotenoids and vitamin $\mathrm{E}$ from their main dietary sources. J Agric Food Chem 2006;54:8749-55.

[33] Goni I, Serrano J, Saura-Calixto F. Bioaccessibility of beta-carotene, lutein, and lycopene from fruits and vegetables. J Agric Food Chem 2006;54:5382-7.

[34] Bohm V, Bitsch R. Intestinal absorption of lycopene from different matrices and interactions to other carotenoids, the lipid status, and the antioxidant capacity of human plasma. Eur J Nutr 1999;38:118-25.

[35] de Pee S, West CE, Permaesih D, Martuti S, Muhilal, Hautvast JG. Orange fruit is more effective than are dark-green, leafy vegetables in increasing serum concentrations of 
retinol and beta-carotene in schoolchildren in Indonesia. Am J Clin Nutr 1998;68:105867.

[36] Zhou JR, Gugger ET, Erdman JW. The crystalline form of carotenes and the food matrix in carrot root decrease the relative bioavailability of beta and alpha-carotene in the ferret model. J Am Coll Nutr 1996;15:84-91.

[37] Gartner C, Stahl W, Sies H. Lycopene is more bioavailable from tomato paste than from fresh tomatoes. Am J Clin Nutr 1997;66:116-22.

[38] Porrini M, Riso P, Testolin G. Absorption of lycopene from single or daily portions of raw and processed tomato. Br J Nutr 1998;80:353-61.

[39] van het Hof KH, deBoer BCJ, Tijburg LBM, Lucius BRHM, Zijp I, West CE, et al. Carotenoid bioavailability in humans from tomatoes processed in different ways determined from the carotenoid response in the triglyceride-rich lipoprotein fraction of plasma after a single consumption and in plasma after four days of consumption. J Nutr 2000;130:1189-96.

[40] Schweiggert RM, Kopec RE, Villalobos-Gutierrez MG, Hogel J, Quesada S, Esquivel P, et al. Carotenoids are more bioavailable from papaya than from tomato and carrot in humans: a randomised cross-over study. Br J Nutr 2014;111:490-8.

[41] De Jesus Ornelas-Paz J, Failla ML, Yahia EM, Gardea-Bejar A. Impact of the stage of ripening and dietary fat on in vitro bioaccessibility of beta-carotene in 'Ataulfo' mango. $\mathrm{J}$ Agric Food Chem 2008;56:1511-6.

[42] Shi J, Le Maguer M. Lycopene in tomatoes: chemical and physical properties affected by food processing. Crit Rev Biotechnol 2000;20:293-334.

[43] Colle I, Lemmens L, Van Buggenhout S, Van Loey A, Hendrickx M. Effect of thermal processing on the degradation, isomerization, and bioaccessibility of lycopene in tomato pulp. J Food Sci 2010;75:C753-9.

[44] Boileau AC, Merchen NR, Wasson K, Atkinson CA, Erdman JW, Jr. Cis-lycopene is more bioavailable than trans-lycopene in vitro and in vivo in lymph-cannulated ferrets. $\mathbf{J}$ Nutr 1999;129:1176-81.

[45] Boileau TW, Boileau AC, Erdman JW. Bioavailability of all-trans and cis-isomers of lycopene. Exp Biol Med (Maywood) 2002;227:914-9.

[46] Brown MJ, Ferruzzi MG, Nguyen ML, Cooper DA, Eldridge AL, Schwartz SJ, et al. Carotenoid bioavailability is higher from salads ingested with full-fat than with fat- 
reduced salad dressings as measured with electrochemical detection. Am J Clin Nutr 2004;80:396-403.

[47] Unlu NZ, Bohn T, Clinton SK, Schwartz SJ. Carotenoid absorption from salad and salsa by humans is enhanced by the addition of avocado or avocado oil. J Nutr 2005;135:4316.

[48] Ribaya-Mercado JD, Maramag CC, Tengco LW, Dolnikowski GG, Blumberg JB, Solon FS. Carotene-rich plant foods ingested with minimal dietary fat enhance the total-body vitamin A pool size in Filipino schoolchildren as assessed by stable-isotope-dilution methodology. Am J Clin Nutr 2007;85:1041-9.

[49] White WS, Zhou Y, Crane A, Dixon P, Quadt F, Flendrig LM. Modeling the dose effects of soybean oil in salad dressing on carotenoid and fat-soluble vitamin bioavailability in salad vegetables. Am J Clin Nutr 2017;106:1041-51.

[50] Borel P, Grolier P, Armand M, Partier A, Lafont H, Lairon D, et al. Carotenoids in biological emulsions: solubility, surface-to-core distribution, and release from lipid droplets. J Lipid Res 1996;37:250-61.

[51] Sy C, Gleize B, Dangles O, Landrier JF, Veyrat CC, Borel P. Effects of physicochemical properties of carotenoids on their bioaccessibility, intestinal cell uptake, and blood and tissue concentrations. Mol Nutr Food Res 2012;56:1385-97.

[52] Tyssandier V, Reboul E, Dumas JF, Bouteloup-Demange C, Armand M, Marcand J, et al. Processing of vegetable-born carotenoids in the human stomach and duodenum. Am $\mathbf{J}$ Physiol Gastrointest Liver Physiol 2003;284:G913-23.

[53] Riedl J, Linseisen J, Hoffmann J, Wolfram G. Some dietary fibers reduce the absorption of carotenoids in women. J Nutr 1999;129:2170-6.

[54] Rock CL, Swendseid ME. Plasma B-carotene response in human after meals supplemented with dietary pectin. Am J Clin Nutr 1992;55:96-9.

[55] Cervantes-Paz B, Ornelas-Paz JJ, Ruiz-Cruz S, Rios-Velasco C, Ibarra-Junquera V, Yahia EM, et al. Effects of pectin on lipid digestion and possible implications for carotenoid bioavailability during pre-absorptive stages: A review. Food Res Int 2017;99:917-27.

[56] Clifton PM, Noakes M, Ross D, Fassoulakis A, Cehun M, Nestel P. High dietary intake of phytosterol esters decreases carotenoids and increases plasma plant sterol levels with no additional cholesterol lowering. J Lipid Res 2004;45:1493-9. 
[57] Richelle M, Enslen M, Hager C, Groux M, Tavazzi I, Godin JP, et al. Both free and esterified plant sterols reduce cholesterol absorption and the bioavailability of betacarotene and alpha-tocopherol in normocholesterolemic humans. Am J Clin Nutr 2004;80:171-7.

[58] Fardet A, Morise A, Kalonji E, Margaritis I, Mariotti F. Influence of phytosterol and phytostanol food supplementation on plasma liposoluble vitamins and provitamin A carotenoid levels in humans: An updated review of the evidence. Crit Rev Food Sci Nutr 2017;57:1906-21.

[59] Reboul E, Thap S, Tourniaire F, Andre M, Juhel C, Morange S, et al. Differential effect of dietary antioxidant classes (carotenoids, polyphenols, vitamin $\mathrm{C}$ and vitamin $\mathrm{E}$ ) on lutein absorption. Br J Nutr 2007;97:440-6.

[60] Abushita AA, Daood HG, Biacs PA. Change in carotenoids and antioxidant vitamins in tomato as a function of varietal and technological factors. J Agric Food Chem 2000;48:2075-81.

[61] Pugliese A, Loizzo MR, Tundis R, O'Callaghan Y, Galvin K, Menichini F, et al. The effect of domestic processing on the content and bioaccessibility of carotenoids from chili peppers (Capsicum species). Food Chem 2013;141:2606-13.

[62] Page D, Van Stratum E, Degrou A, Renard CM. Kinetics of temperature increase during tomato processing modulate the bioaccessibility of lycopene. Food Chem 2012;135:24629.

[63] Edwards AJ, Nguyen CH, You CS, Swanson JE, Emenhiser C, Parker RS. Alpha- and beta-carotene from a commercial puree are more bioavailable to humans than from boiled-mashed carrots, as determined using an extrinsic stable isotope reference method. J Nutr 2002;132:159-67.

[64] van het Hof KH, Gartner C, West CE, Tijburg LBM. Potential of vegetable processing to increase the delivery of carotenoids to man. Int J Vitam Nutr Res 1998;68:366-70.

[65] Seybold C, Frohlich K, Bitsch R, Otto K, Bohm V. Changes in contents of carotenoids and vitamin E during tomato processing. J Agric Food Chem 2004;52:7005-10.

[66] Nguyen ML, Schwartz SJ. Lycopene stability during food processing. Proc Soc Exp Biol Med 1998;218:101-5.

[67] Chen BH, Tang YC. Processing and stability of carotenoid powder from carrot pulp waste. J Agric Food Chem 1998;46:2312-8. 
[68] Minguezmosquera MI, Gandulrojas B. Mechanism and kinetics of carotenoid degradation during the processing of green table olives. J Agric Food Chem 1994;42:1551-4.

[69] Cano MP, Deancos B. Carotenoid and carotenoid ester composition in mango fruit as influenced by processing method. J Agric Food Chem 1994;42:2737-42.

[70] Updike AA, Schwartz SJ. Thermal processing of vegetables increases cis isomers of lutein and zeaxanthin. J Agric Food Chem 2003;51:6184-90.

[71] Rinaldi de Alvarenga JF, Tran C, Hurtado-Barroso S, Martinez-Huelamo M, Illan M, Lamuela-Raventos RM. Home cooking and ingredient synergism improve lycopene isomer production in Sofrito. Food Res Int 2017;99:851-61.

[72] Ghavami A, Coward WA, Bluck LJ. The effect of food preparation on the bioavailability of carotenoids from carrots using intrinsic labelling. Br J Nutr 2012;107:1350-66.

[73] Diaz-Gomez J, Ramos AJ, Zhu C, Martin-Belloso O, Soliva-Fortuny R. Influence of Cooking Conditions on Carotenoid Content and Stability in Porridges Prepared from High-Carotenoid Maize. Plant Foods Hum Nutr 2017;72:113-9.

[74] Berni P, Chitchumroonchokchai C, Canniatti-Brazaca SG, De Moura FF, Failla ML. Impact of genotype and cooking style on the content, retention, and bioacessibility of beta-carotene in biofortified cassava (Manihot esculenta Crantz) conventionally bred in Brazil. J Agric Food Chem 2014;62:6677-86.

[75] Thakkar SK, Huo T, Maziya-Dixon B, Failla ML. Impact of style of processing on retention and bioaccessibility of beta-carotene in cassava (Manihot esculanta, Crantz). $\mathbf{J}$ Agric Food Chem 2009;57:1344-8. 


\section{Légende des figures}

Figure 1 : Localisations intracellulaires et états physiques des caroténoïdes dans différents végétaux. A. Membranes des thylakoïdes retrouvés à l'intérieur des chloroplastes. Les caroténoïdes sont associés aux protéines des photosystèmes. Cette localisation est fréquente dans les légumes à feuilles vertes. B. Chromoplastes cristalloïdes. Les caroténoïdes sont présents sous forme de cristaux solides. Ces chromoplastes sont présents dans les aliments très riches en $\beta$-carotène et en lycopène (respectivement carottes et tomates). $\mathbf{C}$. Chromoplastes tubulaires. Les caroténoïdes sont sous forme de cristaux liquides dans de nombreux fruits. D. Chromoplastes globulaires (plastoglobules). Les caroténoïdes sont solubilisés dans les triglycérides et/ou les phospholipides membranaires. Ces chromoplastes sont fréquents dans les pétales des fleurs mais ils sont plus rares dans les végétaux comestibles. On en trouve dans le mésocarpe comestible du palmier pêche et dans la tomate tangerine (de couleur orange).

Figure 2 : Devenir des caroténoïdes au cours des étapes préalables à leur absorption et facteurs liés à la matrice végétale favorisant ou défavorisant leur biodisponibilité. Les mécanismes par lesquels ces différents facteurs agissent sur la biodisponibilité sont précisés dans le texte. D'autres facteurs liés à la matrice végétale agissent probablement sur la biodisponibilité, e.g. maturation des fruits, polyphénols..., mais seuls les facteurs pour lesquels les preuves sont suffisamment étayées sont mentionnés sur cette figure. 


\section{\begin{tabular}{|c|c|c|}
\hline Lumiere des undakoides \\
\hline
\end{tabular} $\underline{\text { Figure } 1}$}

A

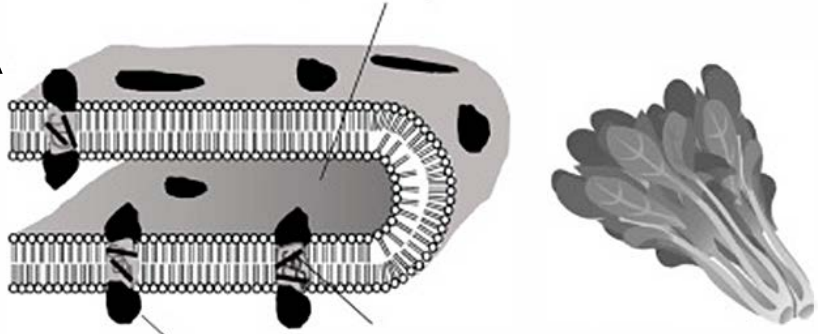

Photosysteme VII

Carot6noî'dos associés à dos protéines

Epinards

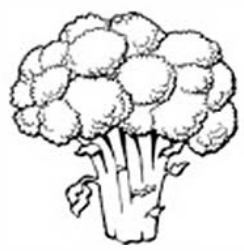

Broccolis

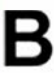

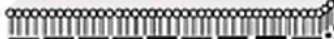

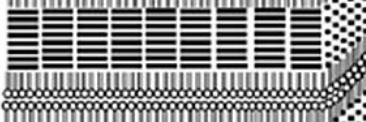

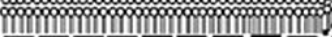

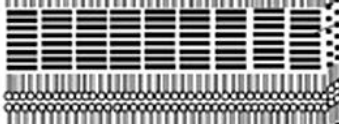

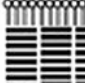

等

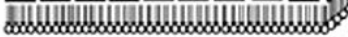

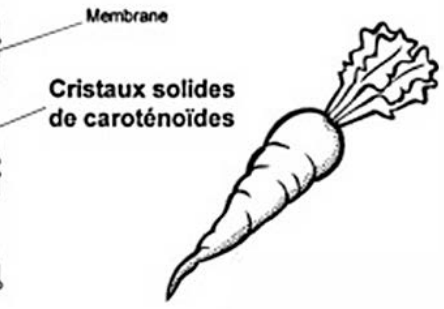

Carottes

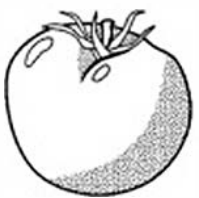

Tomates communes

C

Cristaux liquides do carotônoïdos

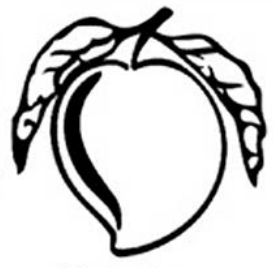

Mangues

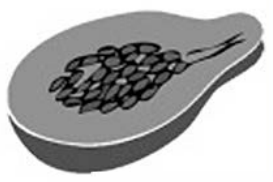

Papayes

\section{D}
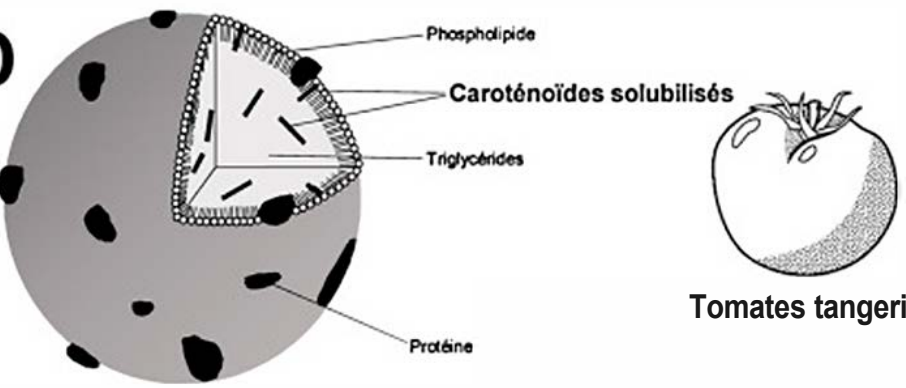

Tomates tangerines 
